\title{
Comportamiento organizacional base para una administración por valores en organizaciones universitarias
}

\section{Behavior organizational base for a management by values in university organizations}

\section{RESUMEN}

Objetivos: Este trabajo pretende aportar hacia un cambio de actitud, porque mucho se habla de globalización; pero no se habla de motivación, moral, ética, valores y compromisos. Métodos: Es oportuno iniciar una nueva etapa de recuperación, principalmente en nuestra autoestima personal, la de nuestra familia, nuestros colaboradores; por eso se presenta resultados del concurso de una prueba tomado a más de 400 estudiantes, en lo que con beneplácito nos indica que los jóvenes tienen espíritu emprendedor y en la universidad se desperdician por no cultivar $\mathrm{u}$ orientar a que ellos mismos se descubran. Resultados: Se establecen criterios completos sobre, comportamiento organizacional, su desarrollo y el alcance de nuestras culturas. Lo que se trata es de dar un reforzamiento ético a la globalización económica ya que el "imperialismo" dejo crisis axiológica centrado totalmente en el materialismo pragmático. Conclusiones: La administración por valores tiene mayores resultados positivos en la gestión institucional que la administración por objetivos, debido a que las personas se desenvuelven satisfechos en lo que realizan "sin presión de un control o evaluación".

Palabras clave: Comportamiento organizacional, administración, valores, universidad.

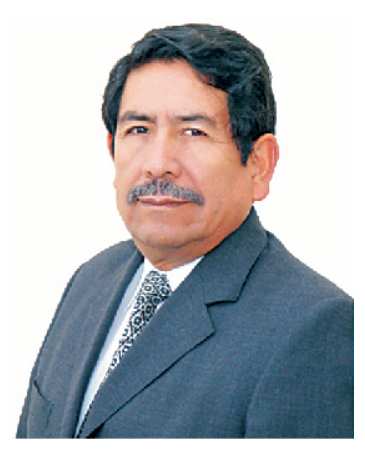

Esau Caro Meza

Universidad Continental de Ciencias e Ingeniería

\section{ABSTRACT}

Objectives: This work seeks to contribute toward a change of attitude, because globalization is very discussed theme but motivation, moral, ethics, values, and commitments themes are not discussed. Methods: It is opportune to begin a new recovery stage, mainly in our personal self-esteem, of our family, our collaborators; for that reason it is show the results of the competition in a test taken for more than 400 students, in what indicates us with approval that the youths have venturesome spirit and at the university they are wasted because those are not cultivated or guided to discover themselves. Results: Enterie judgments about organizational behavior, its development and the reach of our cultures and stablished. What is it

1 Doctor en Administración, Universidad Nacional Federico Villarreal. Ex Rector de la Universidad Nacional del Centro del Perú, Rector de la Universidad Continental de Ciencias e Ingeniería. 
comes to give is an ethical strengthening to the economic globalization, since "imperialism" left the axiological crisis focused enterely on pragmatic materialism. Conclusions: The management by values has more positive results in the institutional administration than the management for objectives, because people are unfold satisfied in what they carry out "without pressure of a control or an evaluation."

Key words: behavior organizational, management, values, university.

\section{INTRODUCCIÓN}

De los resultados obtenidos se puede establecer criterios complejos sobre, comportamiento organizacional, su desarrollo y el alcance de nuevas culturas, tratamos de delinear los procesos de una administración por valores como una alternativa de cambio para lograr una mayor productividad de los miembros de la comunidad universitaria, ya que por un predominio de antivalores y contracultura no alcanza su verdadero desarrollo y cumplimiento de sus funciones de servicio a la comunidad y a las empresas, lo que se trata es de dar un reforzamiento ético a la globalización económica ya que el "imperialismo" dejó una crisis axiológica centrado totalmente en el materialismo pragmático (1) El error más frecuente y lamentable a la hora de reformular la visión, misión y valores operativos de la empresa, es el de publicarlo en un bonito formato y después no hacer absolutamente nada para evaluar y para recompensar su asimilación y cumplimiento (2).

No se cuenta con la presencia de trabajos de investigación o experiencias sobre una administración por valores en nuestro país, y más aún en organizaciones académicas públicas. Internacionalmente si existen algunas especialmente en Estados Unidos de América, quienes consideran una opción para mejorar la eficacia de las organizaciones.

Ahora nuestro país de debate en una crisis, especialmente las universidades, instituciones que deberían ser líderes en la soluciones nacionales, sin embargo, estas se encuentran divorciadas del concepto común de trabajo integrado o trabajo en equipo, la sociedad no cree en la universidad, por lo tanto, se puede afirmar que es necesaria la realización del levantamiento de un sistema de información sobre el comportamiento organizacional actual de la Universidad Peruana, para establecer cuáles son las actitudes, valores y creencias con que vienes trabajando en base al cual buscar un desarrollo integrado para lograr una nueva cultura organizacional universitaria y trabajar en base a una nueva administración por valores y así ubicar al sistema universitario como alternativa a la solución de los problemas nacionales.

En qué medida el desarrollo de una cultura organizacional nueva influye positivamente en el establecimiento de una administración por valores para lograr el desarrollo de la organización universitaria.

Mediante un programa adecuado de cultura organizacional se logrará mejorar la administración universitaria adaptando a los recursos humanos a un nuevo sistema cultural de desarrollo organizacional de las universidades.

Fomentar la cultura organizacional de la institución promoviendo la iniciativa y creatividad para el progreso mediante una gestión adicional, la administración por valores de tal manera que su utilización sistemática se establezca a partir de un modelamiento organizacional.

\section{MATERIAL Y MEETODOS}

El estudio a realizarse para lograr una administración por valores en el Sistema de la Universidad Peruana y en forma especial en la UNCP estableciendo un comportamiento organizacional en base a una cultura nueva, es una investigación Aplicada en merito a que se tomaron conocimientos y teorías de las ciencias administrativas, la Psicología y la Sociología para ser usado en la gestión institucional y logre su desarrollo determinando acciones concretas en el tiempo y buscando una cultura organizacional en la comunidad universitaria para establecer nuevas escalas de valores y romper esquemas y paradigmas arcaicos y anacrónicos. 


\section{Universo}

Es el Sistema de la Universidad Peruana, considerando las universidades nacionales y privadas del Perú.

\section{Población}

La población de investigación fueron las universidades ubicadas en el departamento de Junín, especialmente la Universidad Nacional del Centro del Perú, comprendida entre su personal docente, administrativo y estudiantes, que viene a ser una población de 10000 personas.

\section{Muestra}

La muestra seleccionada fue del tipo no probabilístico y estuvo distribuido de la siguiente manera:

Ecuaciones de Nivel del Modelo Dinámico de Administración por Valores:

1. APRENDIZAJE $(t)=\operatorname{APRENDIZAJE}(t-d t)+$ (FLUJO DE APRENDIZAJE DESAPRENDIZAJE) $* d t$

2. $C A M B I O(t)=C A M B I O(t-d t)+F L U J O \_D E$ CAMBIO FLUJO_DE_APRENDIZAJE) ${ }^{*} d t$

3. DESEMPEÑO $(t)=\operatorname{DESEMPEÑO}(t-d t)+$ (FLUJO_DE_DESEMPEÑO - BAJA_DEL DESEMPEÑO) ${ }^{*} d t$

4. MOTIVACIÓN $(t)=$ MOTIVACIÓN $(t-d t)+$ (NECESIDADES_SATISFECHAS + FLUJO_DE _MOTIVACIÓN) * dt

5. POLARIZACIÓN $(t)=$ POLARIZACIÓN $(t-d t)+$ (FLUJO DE POLARIZACIÓN - DISMINU CIÓN_DE_POLARIZACIÓN) * dt

6. RESOLUCION DE PROBLEMAS( $t)=$ RESOLUCION_DE_PROBLEMAS $(t \quad d t)+$ (FLUJO_DE_RESOLUCIÓN_DE_PROBLEMAS SOLUCIÓN) ${ }^{*} d t$

7. $\operatorname{RESULTADOS}(t)=\operatorname{RESULTADOS}(t-d t)+$
(FLUJO_DE_RESULTADOS - FLUJO_DE_ CAMBIO) $* \overline{d t}$

Número de integrantes de la muestra: 400 personas.

\section{RESULTADOS}

Como resultado de los trabajos de taller y análisis estadístico podemos asegurar que la herramienta de los líderes es la persuasión y el poder, considerando que el instrumento del poder son las reglas, y que la persuasión necesita principio y coherencia, esto se contagia a través de la razón o de la emoción.

El estudio de los actos o conductas y actitudes de los miembros: docentes, administrativos y estudiantes, nos han demostrado una productividad baja; docentes indiferentes, administrativos insatisfechos y docentes desmotivados y sin metas fijas, el ausentismo es elevado y las rotaciones de personal muy constante.

La Universidad Nacional del Centro del Perú después de varios años de trabajo continuo, de persuasión y con muchos talleres ha logrado establecer su visión, misión y su escala de valores, internalizando en cada trabajador y estudiante el compromiso con la responsabilidad que tienen.

El comportamiento del personal en la universidad se ha establecido en 3 niveles: el individuo se encontró diferencias y poca voluntad porque estaban en puestos no compatibles con su personalidad y función. A nivel de grupo entenderlo fue más complicado, poca coordinación, participación mínima y trabajo en equipo nulo. Y a nivel de sistema se tenía una estructura orgánica arcaica con procesos no funcionales. Con u n programa de persuasión e incentivos se ha logrado mejorar el desempeño y satisfacción, aumentar su participación en la solución de problemas.

Se ha cambiado la estructura orgánica de la universidad, sus procesos y procedimientos administrativos estableciendo sistemas de recompensas e incentivos, se ha diseñado puestos y la cultura con escala de valores. Pequeños cambios que a la fecha han dado como resultado mayor productividad. 
La administración por valores establecida en la universidad ha alcanzado nuevos niveles de éxito organizacional, debido a que las organizaciones son dinámicas, se caracterizan por el cambio constante en tecnología, cultura, social, económico y personal, ha disminuido los efectos de ansiedad, el estrés, inseguridad y presión en los trabajadores.

La administración por valores en base al compromiso han dado a la universidad una solución práctica para lograr estabilidad, continuidad y crecimiento a medida de los retos del entorno.

Con la administración por valores tenemos una guía para implementar en la universidad un enfoque basado en valores y compromiso con la institución. Por la tanto la aplicación de la administración por valores es un futuro prometedor, haciendo que todos los integrantes de la organización quedan satisfechos en su realización y en el servicio a los demás.

El comportamiento organizacional contribuye a la ejecución exitosa de la administración por valores o contrariamente es un factor que la obstaculiza. Por eso las organizaciones que cambian de estrategias, visión, misión y sus estructuras fracasan debido a que el personal no apoya en nuevo enfoque.

La universidad es una organización influenciada por su pasado, la sociedad y las contingencias, elementos componentes de su cultura organizacional. Es necesario internalizar sus valores a base de persuasión, así como su visión para que se pueda practicar una administración porvalores.

\section{DISCUSIÓN}

La administración versa en cuatro campos muy importantes. Sin embargo esto no está dando resultados, debido a que todo se sujeta al control, el trabajador cumple o simula en cumplir solo por el control. Las organizaciones podrían llenar este vacío desde sus filas internas si supieran como desarrollar sus verdaderos líderes potenciales. No hay escases de talentos.
Remendar y ajusta no resolverán el problema fundamental. Para resolver el problema debe atacarse de raíz. Analizar el comportamiento de cada uno de sus trabajadores lo que se refleja mediante la actitud personal y corporativa, si esto fuera positivo se podrá engendrar el compromiso en la comunidad.

El trabajador debe comprender que la actitud es muy importante pero no lo es todo, porque debemos entender que la actitud no sólo son cuotas de momento sino también potencialmente cambiadores de vida, porque la gente que tiene éxito no tiene menos desafíos que los demás. Es necesario saber que los exitosos experimentan más "fracasos", vencen más problemas, manejan más cambios y trata con más posibilidades y desánimo y temor que aquellos que no tienen éxito.

La actitud de estos y aquellos diferencian en el comportamiento de la organización permitiendo una administración no sujeta al control sino al compromiso con la práctica de valores.

Observamos que el comportamiento de la organización son positivas permitiendo una administración por valores si se respeta algunos derechos del trabajador como: Derechos a sentirse necesitados, sentirse a ser involucrados, a una relación contractual a influir en su propio destino, a apelar y sobre el derecho a comprometerse.

De acuerdo a los resultados obtenidos, se observa que la clasificación de los valores instrumentales planteados por Garcia y Dolan (2), son percibidos como valores sumamente importantes para incrementar la productividad tanto para los docentes-investigadores universitarios con experiencia empresarial, como los profesionales-estudiantes de maestría y los trabajadores de empresas. Por lo que esta clasificación puede servir de referencia para iniciar investigaciones sobre los valores que deben prevalecer en las PYME's para lograr incrementar la productividad. 


\section{REFERENCIAS BIBLIOGRAFCAS}

1. Blanchard K, O Connor M. Administración por valores, cómo lograr el éxito organizacional y personal mediante el compromiso con una misión y unos valores compartidos. 1 ra Edición. Bogota: Ed. Norma; 1997.

2. García S, Dolan S. La dirección por valores. El cambio más allá de la dirección por objetivos. Madrid: Edit. McGraw-Hill Interamericana; 1997.

3. Darwin C. Enciclopedia Microsoft Encarta. Microsoft Corporation. Versión electrónica. En: http://www.argentinawarez.com/programas-gratis/1353417micorsoft-encarta-2009-actualizada-2011-espanol-dvd.html. 2009.

4. Naisbitt J, Aburdene P. Megatendencias. 1ra Edición. Madrid: Ed. Norma; 2000.

5. Suarez Z, Ignacio G. Harvard Business Review; 1ra Edición. New York: Ed. Agusto S.A.; 2000.

Correo electrónico:

ecaro@continental.edu.pe 